\title{
New sufficient conditions for the asymptotic stability of discrete time-delay systems
}

\author{
P Sangapate
}

Correspondence: prisayarat@mju.ac. th

Department of Mathematics, Maejo University, Chiangmai, 50290,

Thailand

\begin{abstract}
This paper is concerned with asymptotic stability of switched discrete time-delay systems. The system to be considered is subject to interval time-varying delays, which allows the delay to be a fast time-varying function and the lower bound is not restricted to zero. Based on the discrete Lyapunov functional, a switching rule for the asymptotic stability for the system is designed via linear matrix inequalities. Numerical example is included to illustrate the effectiveness of the result.
\end{abstract}

Keywords: Switching design, discrete system, asymptotic stability, Lyapunov function, linear matrix inequality

\section{Introduction}

As an important class of hybrid systems, switched systems arise in many practical processes that cannot be described by exclusively continuous or exclusively discrete models, such as manufacturing, communication networks, automotive engineering control and chemical processes (see, e.g., [1-3] and the references therein). On the other hand, time-delay phenomena are very common in practical systems. A switched system with time-delay individual subsystems is called a switched time-delay system; in particular, when the subsystems are linear, it is then called a switched time-delay linear system. During the last decades, the stability analysis of switched linear continuous/discrete time-delay systems has attracted a lot of attention [4-18]. The main approach for stability analysis relies on the use of Lyapunov-Krasovskii functionals and linear matrix inequlity (LMI) approach for constructing a common Lyapunov function [19-24]. Although many important results have been obtained for switched linear continuoustime systems, there are few results concerning the stability of switched linear discrete systems with time-varying delays. It was shown in $[5,7,11]$ that when all subsystems are asymptotically stable, the switching system is asymptotically stable under an arbitrary switching rule. The asymptotic stability for switching linear discrete time-delay systems has been studied in [10], but the result was limited to constant delays. In [11], a class of switching signals has been identified for the considered switched discretetime delay systems to be stable under the average dwell time scheme.

This paper studies asymptotic stability problem for switched linear discrete systems with interval time-varying delays. Specifically, our goal is to develop a constructive way to design switching rule to asymptotically stabilize the system. By using improved Lyapunov-Krasovskii functionals combined with LMIs technique, we propose new criteria

(c) 2012 Sangapate; licensee Springer. This is an Open Access article distributed under the terms of the Creative Commons Attribution License (http://creativecommons.org/licenses/by/2.0), which permits unrestricted use, distribution, and reproduction in any medium, provided the original work is properly cited. 
for the asymptotic stability of the system. Compared to the existing results, our result has its own advantages. First, the time delay is assumed to be a time-varying function belonging to a given interval, which means that the lower and upper bounds for the time-varying delay are available, the delay function is bounded but not restricted to zero. Second, the approach allows us to design the switching rule for stbility in terms of of LMIs, which can be solvable by utilizing Matlab's LMI Control Toolbox available in the literature to date.

The paper is organized as follows: Section 2 presents definitions and some wellknown technical propositions needed for the proof of the main results. Switching rule for the asymptotic stability is presented in Section 3. Numerical example of the result is given in Section 4.

\section{Preliminaries}

The following notations will be used throughout this paper. $R^{+}$denotes the set of all real non-negative numbers; $R^{n}$ denotes the $n$-dimensional space with the scalar product of two vectors $\langle x, y\rangle$ or $x^{T} y ; R^{n \times r}$ denotes the space of all matrices of $(n \times r)$ dimension. $A^{T}$ denotes the transpose of $A$; a matrix $A$ is symmetric if $A=A^{T}$.

Matrix $A$ is semi-positive definite $(A \geq 0)$ if $\langle A x, x\rangle \geq 0$, for all $x \in R^{n} ; A$ is positive definite $(A>0)$ if $\langle A x, x\rangle>0$ for all $x \neq 0 ; A \geq B$ means $A-B \geq 0$. $\lambda(A)$ denotes the set of all eigenvalues of $A ; \lambda_{\min }(A)=\min \{\operatorname{Re} \lambda: \lambda \in \lambda(A)\}$.

Consider a discrete systems with interval time-varying delay of the form

$$
\begin{aligned}
x(k+1) & =A_{\gamma} x(k)+B_{\gamma} x(k-d(k)), k=0,1,2, \ldots \\
x(k) & =v_{k}, k=-d_{2},-d_{2}+1, \ldots, 0,
\end{aligned}
$$

where $x(k) \in R^{n}$ is the state, $\gamma():. R^{n} \rightarrow N:=\{1,2, \ldots, N\}$ is the switching rule, which is a function depending on the state at each time and will be designed. A switching function is a rule which determines a switching sequence for a given switching system. Moreover, $\gamma(x(k))=i$ implies that the system realization is chosen as the $i^{\text {th }}$ system, $i=$ $1,2, \ldots, N$. It is seen that the system (1) can be viewed as an autonomous switched system in which the effective subsystem changes when the state $x(k)$ hits predefined boundaries. $A_{i}, B_{i} i=1,2, \ldots, N$ are given constant matrices. The time-varying function $d(k)$ satisfies the following condition:

$$
0<d_{1} \leq d(k) \leq d_{2}, \quad \forall k=0,1,2, \ldots
$$

Remark 1 It is worth noting that the time delay is a time-varying function belonging to a given interval, in which the lower bound of delay is not restricted to zero.

Definition 1 The switched system (1) is asymptotically stable if there exists a switching function $\gamma($.$) such that the zero solution of the system is asymptotically stable.$

Definition 2 The system of matrices $\left\{V_{i}\right\}, i=1,2, \ldots, N$, is said to be strictly complete if for every $x \in R^{n} \backslash\{0\}$ there is $i \in\{1,2, \ldots, N\}$ such that $x^{T} J_{i} x<0$.

It is easy to see that the system $\left\{J_{i}\right\}$ is strictly complete if and only if

$$
\bigcup_{i=1}^{N} \alpha_{i}=R^{n} \backslash\{0\}
$$


where

$$
\alpha_{i}=\left\{x \in R^{n}: \quad x^{T} J_{i} x<0\right\}, i=1,2, \ldots, N .
$$

Proposition 1 [12] The system $\left\{J_{i}\right\}, i=1,2, \ldots, N$, is strictly complete if there exist $\delta_{i} \geq$ $0, i=1,2, \ldots, N, \sum_{i=1}^{N} \delta_{i}>$ osuch that

$$
\sum_{i=1}^{N} \delta_{i} J_{i}<0
$$

If $N=2$ then the above condition is also necessary for the strict completeness.

\section{Main results}

Let us set

$$
\begin{aligned}
& \mathcal{W}_{i}(P, Q, R)=\left(\begin{array}{ccc}
Q-P & R^{T}-A_{i}^{T} R-R^{T} B_{i} \\
R-R^{T} A_{i} P+R+R^{T} & -R^{T} B_{i} \\
-B_{i}^{T} R & -B_{i}^{T} R & -Q
\end{array}\right), \\
& J_{i}(P, Q)=\left(d_{2}-d_{1}\right) Q-R^{T} A_{i}-A_{i}^{T} R, \quad \lambda_{1}=\lambda_{\min }(P), \\
& \alpha_{i}=\left\{x \in R^{n}: \quad x^{T} J_{i}(R, Q) x<0\right\}, i=1,2, \ldots, N, \\
& \bar{\alpha}_{1}=\alpha_{1}, \bar{\alpha}_{i}=\alpha_{i} \backslash \bigcup_{j=1}^{i-1} \bar{\alpha}_{j}, i=2,3, \ldots, N .
\end{aligned}
$$

The main result of this paper is summarized in the following theorem.

Theorem 1 The switched system (1) is asymptotically stable if there exist symmetric positive definite matrices $P>0, Q>0$ and matrix $R$ satisfying the following conditions $(i) \exists \delta_{i} \geq 0, i=1,2, \ldots, N, \sum_{i=1}^{N} \delta_{i}>0: \sum_{\mathrm{i}=1}^{N} \delta_{\mathrm{i}} J_{i}(R, Q)<0$.

(ii) $\mathcal{W}_{i}(P, Q, R)<0, \quad i=1,2, \ldots, N$.

The switching rule is chosen as $\gamma(x(k))=i$, whenever $x(k) \in \bar{\alpha}_{i}$.

Proof. Consider the following Lyapunov-Krasovskii functional for any $i$ th system (1)

$$
V_{(k)}=V_{1}(k)+V_{2}(k)+V_{3}(k),
$$

where

$$
\begin{gathered}
V_{1}(k)=x^{T}(k) P x(k), \quad V_{2}(k)=\sum_{i=k-d(k)}^{k-1} x^{T}(i) Q x(i), \\
V_{3}(k)=\sum_{j=-d_{2}+2}^{-d_{1}+1} \sum_{l=k+j}^{k-1} x^{T}(l) Q x(l),
\end{gathered}
$$

We can verify that

$$
\lambda_{1}\|x(k)\|^{2} \leq V(k) .
$$

Let us set $\xi(k)=[x(k) x(k+1) x(k-d(k))]^{T}$, and

$$
H=\left(\begin{array}{lll}
0 & 0 & 0 \\
0 & P & 0 \\
0 & 0 & 0
\end{array}\right), \quad G=\left(\begin{array}{ccc}
P & 0 & 0 \\
R & R & 0 \\
R & 0 & I
\end{array}\right) .
$$


Then, the difference of $V_{1}(k)$ along the solution of the system is given by

$$
\begin{aligned}
\Delta V_{1}(k) & =x^{T}(k+1) \operatorname{Px}(k+1)-x^{T}(k) \operatorname{Px}(k) \\
& =\xi^{T}(k) H \xi(k)-2 \xi^{T}(k) G^{T}\left(\begin{array}{c}
0.5 x(k) \\
0 \\
0
\end{array}\right) .
\end{aligned}
$$

because of

$$
\xi^{T}(k) H \xi(k)=x(k+1) P x(k+1) .
$$

Using the expression of system (1)

$$
0=-x(k+1)+A_{i}(k)+B_{i} x(k-d(k)),
$$

we have

$$
\begin{aligned}
& -2 \xi^{T}(k) G^{T}\left(-x(k+1)+\underset{0}{0.5 x(k)} \underset{0}{A}(k)+B_{i} x(k-d(k))\right) \xi(k) \\
& =-\xi^{T}(k) G^{T}\left(\begin{array}{ccc}
0.5 I & 0 & 0 \\
A_{i} & -I & B_{i} \\
0 & 0 & 0
\end{array}\right) \xi(k)-\xi^{T}(k)\left(\begin{array}{ccc}
0.5 I & A_{i}^{T} & 0 \\
0 & -I & 0 \\
0 & B_{i}^{T} & 0
\end{array}\right) G \xi(k) .
\end{aligned}
$$

Therefore, from (3) it follows that

$$
\Delta V_{1}(k)=\xi^{T}(k) W_{i} \xi(k),
$$

where

$$
W_{i}=\left(\begin{array}{lll}
0 & 0 & 0 \\
0 & P & 0 \\
0 & 0 & 0
\end{array}\right)-G^{T}\left(\begin{array}{ccc}
0.5 I & 0 & 0 \\
A_{i} & -I & B_{i} \\
0 & 0 & 0
\end{array}\right)-\left(\begin{array}{ccc}
0.5 I & A_{i}^{T} & 0 \\
0 & -I & 0 \\
0 & B_{i}^{T} & 0
\end{array}\right) G
$$

The difference of $V_{2}(k)$ is given by

$$
\begin{aligned}
\Delta V_{2}(k)= & \sum_{i=k+1-d(k+1)}^{k} x^{T}(i) Q x(i)-\sum_{i=k-d(k)}^{k-1} x^{T}(i) Q x(i) \\
= & \sum_{i=k+1-d(k+1)}^{k-d_{1}} x^{T}(i) Q x(i)+x^{T}(k) Q x(k)-x^{T}(k-d(k)) Q x(k-d(k)) \\
& +\sum_{i=k+1-d_{1}}^{k-1} x^{T}(i) Q x(i)-\sum_{i=k+1-d(k)}^{k-1} x^{T}(i) Q x(i) .
\end{aligned}
$$

Since $d(k) \geq d_{1}$ we have

$$
\sum_{i=k+1-d_{1}}^{k-1} x^{T}(i) Q x(i)-\sum_{i=k+1-d(k)}^{k-1} x^{T}(i) Q x(i) \leq 0,
$$

and hence from (6) we have

$$
\Delta V_{2}(k) \leq \sum_{i=k+1-d(k+1)}^{k-d_{1}} x^{T}(i) Q x(i)+x^{T}(k) Q x(k)-x^{T}(k-d(k)) Q x(k-d(k)) .
$$


The difference of $V_{3}(k)$ is given by

$$
\begin{aligned}
\Delta V_{3}(k) & =\sum_{j=-d_{2}+2}^{-d_{1}+1} \sum_{l=k+j+1}^{k} x^{T}(l) Q x(l)-\sum_{j=-d_{2}+2}^{-d_{1}+1} \sum_{l=k+j}^{k-1} x^{T}(l) Q x(l) \\
& =\sum_{j=-d_{2}+2}^{-d_{1}+1}\left[\sum_{l=k+j}^{k-1} x^{T}(l) Q x(l)+x^{T}(k) Q(\xi) x(k)\right. \\
& \left.-\sum_{l=k+j}^{k-1} x^{T}(l) Q x(l)-x^{T}(k+j-1) Q x(k+j-1)\right] \\
& =\sum_{j=-d_{2}+2}^{-d_{1}+1}\left[x^{T}(k) Q x(k)-x^{T}(k+j-1) Q x(k+j-1)\right] \\
& =\left(d_{2}-d_{1}\right) x^{T}(k) Q x(k)-\sum_{j=k+1-d_{2}}^{k-d_{1}} x^{T}(j) Q x(j) .
\end{aligned}
$$

Since $d(k) \leq d_{2}$, and

$$
\sum_{i=k+1-d(k+1)}^{k-d_{1}} x^{T}(i) Q x(i)-\sum_{i=k+1-d_{2}}^{k-d_{1}} x^{T}(i) Q x(i) \leq 0,
$$

we obtain from (7) and (8) that

$$
\Delta V_{2}(k)+\Delta V_{3}(k) \leq\left(d_{2}-d_{1}+1\right) x^{T}(k) Q x(k)-x^{T}(k-d(k)) Q x(k-d(k)) .
$$

Therefore, combining the inequalities (5), (9) gives

$$
\Delta V(k) \leq x^{T}(k) J_{i}(P, Q) x(k)+\xi^{T}(k) W_{i}(P, Q, R) \xi(k),
$$

where

$$
W_{i}(P, Q, R)=\left(\begin{array}{ccc}
Q-P & R^{T}-A_{i}^{T} R-R^{T} B_{i} \\
R-R^{T} A_{i} P+R+R^{T} & -R^{T} B_{i} \\
-B_{i}^{T} R & -B_{i}^{T} R & -Q
\end{array}\right) .
$$

Therefore, we finally obtain from (10) and the condition (ii) that

$$
\Delta V(k)<x^{T}(k) J_{i}(R, Q) x(k), \quad \forall i=1,2, \ldots, N, k=0,1,2, \ldots .
$$

We now apply the condition (i) and Proposition 1 , the system $J_{i}(R, Q)$ is strictly complete, and the sets $\alpha_{i}$ and $\bar{\alpha}_{i}$ by (2) are well defined such that

$$
\begin{gathered}
\bigcup_{i=1}^{N} \alpha_{i}=R^{n} \backslash\{0\}, \\
\bigcup_{i=1}^{N} \bar{\alpha}_{i}=R^{n} \backslash\{0\}, \quad \bar{\alpha}_{i} \cap \bar{\alpha}_{j}=\emptyset, i \neq j .
\end{gathered}
$$

Therefore, for any $x(k) \in R^{n}, k=1,2, \ldots$, there exists $i \in\{1,2, \ldots, N\}$ such that $x(k) \in \bar{\alpha}_{i}$. By choosing switching rule as $\gamma(x(k))=i$ whenever $x(k) \in \bar{\alpha}_{i}$, from the condition (10) we have

$$
\Delta V(k) \leq x^{T}(k) J_{i}(R, Q) x(k)<0, k=1,2, \ldots,
$$


which, combining the condition (3) and the Lyapunov stability theorem [12], concludes the proof of the theorem.

Remark 2 Note that the resuts proposed in [4-6] for switching systems to be asymptotically stable under an arbitrary switching rule. The asymptotic stability for switching linear discrete time-delay systems studied in [9] was limited to constant delays. In [10], a class of switching signals has been identified for the considered switched discretetime delay systems to be stable under the averaged well time scheme.

\section{Numerical example}

Example 1 Consider the switched discrete-time system (1), where $d_{1}=1, d_{2}=4$ and

$$
\begin{gathered}
\left(A_{1}, B_{1}\right)=\left(\left[\begin{array}{cc}
-0.1 & 0.01 \\
0.02 & -0.2
\end{array}\right],\left[\begin{array}{cc}
-0.1 & 0.01 \\
0.02 & -0.3
\end{array}\right]\right), \\
\left(A_{2}, B_{2}\right)=\left(\left[\begin{array}{cc}
1 & 0.2 \\
0.1 & 2
\end{array}\right],\left[\begin{array}{cc}
0.1 & 0.02 \\
0.01 & 0.2
\end{array}\right]\right) .
\end{gathered}
$$

By LMI toolbox of Matlab, we find that the conditions (i), (ii) of Theorem 1 are satisfied with $\delta_{1}=0.1, \delta_{2}=0.2$ and

$$
P=\left[\begin{array}{ll}
0.7339 & 0.0006 \\
0.0006 & 0.8383
\end{array}\right], Q=\left[\begin{array}{ll}
0.2817 & 0.0138 \\
0.0138 & 0.3773
\end{array}\right], R=\left[\begin{array}{cc}
-0.9091 & -0.1261 \\
0.1311 & -0.9935
\end{array}\right] .
$$

In this case, we have

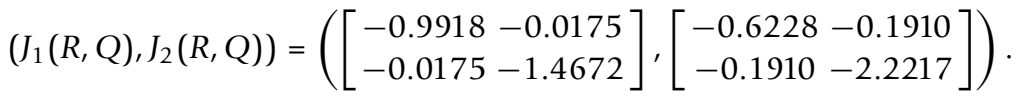

Moreover, the sum

$$
\delta_{1} J_{1}(R, Q)+\delta_{2} J_{2}(R, Q)=\left[\begin{array}{ll}
-0.2238 & -0.0400 \\
-0.0400 & -0.5911
\end{array}\right]
$$

is negative definite; i.e. the first entry in the first row and the first column -0.22380 is negative and the determinant of the matrix is positive. The sets $\alpha_{1}$ and $\alpha_{2}$ in Figure 1 and Figure 2 are given as

$$
\begin{aligned}
& \alpha_{1}=\left\{\left(x_{1}, x_{2}\right):-0.9918 x_{1}^{2}-0.035 x_{1} x_{2}-0.1 .4672 x_{2}^{2}<0\right\}, \\
& \alpha_{2}=\left\{\left(x_{1}, x_{2}\right): 0.6228 x_{1}^{2}+0.382 x_{1} x_{2}+2.2217 x_{2}^{2}>0\right\} .
\end{aligned}
$$

Obviously, the union of these sets is equal to $R^{2} \backslash\{0\}$. The switching regions are defined as

$$
\begin{gathered}
\bar{\alpha}_{1}=\left\{\left(x_{1}, x_{2}\right):-0.9918 x_{1}^{2}-0.035 x_{1} x_{2}-0.1 .4672 x_{2}^{2}<0\right\} \\
\bar{\alpha}_{2}=\alpha_{2} \backslash \bar{\alpha}_{1}
\end{gathered}
$$

By Theorem 1 the system is asymptotically stable and the switching rule is chosen as $\gamma(x(k))=i$ whenever $x(k) \in \bar{\alpha}_{i}$.

\section{Conclusion}

This paper has proposed a switching design for the asymptotic stability of switched linear discrete-time systems with interval time-varying delays. Based on the discrete 

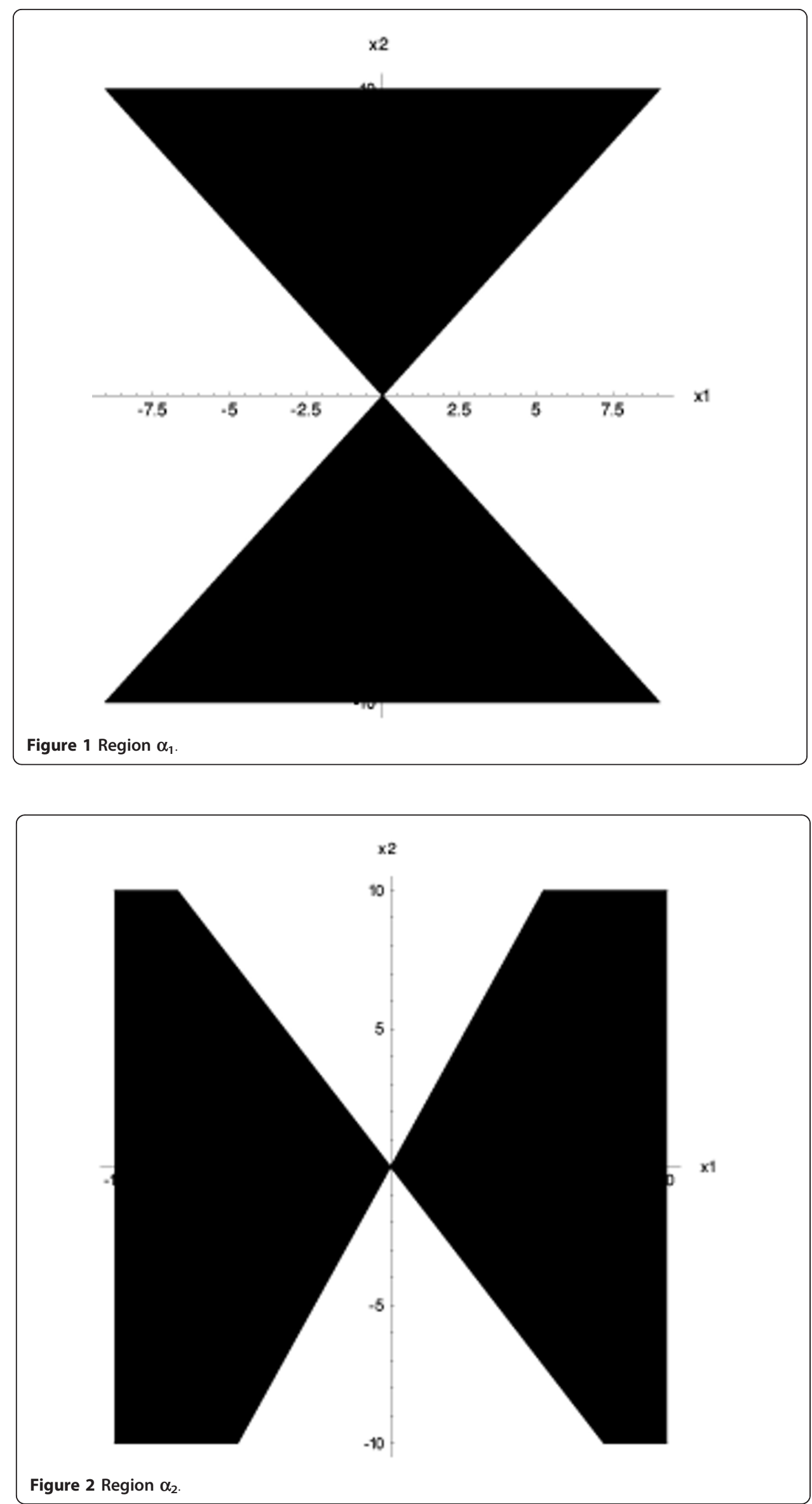
Lyapunov functional, a switching rule for the asymptotic stability for the system is designed via linear matrix inequalities.

\section{Acknowledgements}

This work was supported by the Thai Research Fund Grant, the Higher Education Commission and Faculty of Science, Maejo University, Thailand.

\section{Authors' contributions}

The authors declare that the study was realized in collaboration with the same responsibility. All authors read and approved the final manuscript.

\section{Competing interests}

The author declares that they have no competing interests.

Received: 26 September 2011 Accepted: 8 March 2012 Published: 8 March 2012

\section{References}

1. Liberzon, D, Morse, AS: Basic problems in stability and design of switched systems. IEEE Control Syst Mag. 19, 57-70 (1999)

2. Savkin, AV, Evans, RJ: Hybrid Dynamical Systems: Controller and Sensor Switching Problems. Springer. New York (2001)

3. Ratchagit, K: Asymptotic stability of nonlinear delay-difference system via matrix inequalities and application. International Journal of Computational Methods. 389-397 (2009)

4. Sun, Z, Ge, SS: Switched Linear Systems: Control and Design. Springer. London (2005)

5. Phat, VN, Kongtham, Y, Ratchagit, K: LMI approach to exponential stability of linear systems with interval time-varying delays. Linear Algebra Appl. 436, 243-251 (2012). doi:10.1016/j.laa.2011.07.016

6. Gao, F, Zhong, S, Gao, X: Delay-dependent stability of a type of linear switching systems with discrete and distributed time delays. Appl Math Computation. 196, 24-39 (2008). doi:10.1016/j.amc.2007.05.053

7. Lien, $\mathrm{CH}, \mathrm{Yu}, \mathrm{KW}$, Chung, YJ, Lin, YF, Chung, LY, Chen, JD: Exponential stability analysis for uncertain switched neutral systems with interval-time-varying state delay. Nonlinear Analysis: Hybrid systems. 3, 334-342 (2009). doi:10.1016/j. nahs.2009.02.010

8. Phat, VN, Ratchagit, K: Stability and stabilization of switched linear discrete-time systems with interval time-varying delay. Nonlinear Analysis: Hybrid Systems. 5, 605-612 (2011). doi:10.1016/j.nahs.2011.05.006

9. Ratchagit, K, Phat, VN: Stability criterion for discrete-time systems. Journal of Inequalities and Applications. 10, 1-6 (2010)

10. Xie, G, Wang, L: Quadratic stability and stabilization of discrete-time switched systems with state delay. Atlantics. pp. 3235-3240.Proc of the IEEE Conference on Decision and Control (2004)

11. Boyd, S, Ghaoui, LE, Feron, E, Balakrishnan, V: Linear Matrix Inequalities in System and Control Theory. SIAM. Philadelphia (1994)

12. Ji, DH, Park, JH, Yoo, WJ, Won, SC: Robust memory state feedback model predictive control for discrete-time uncertain state delayed systems. Appl Math Computation. 215, 2035-2044 (2009). doi:10.1016/j.amc.2009.07.052

13. Zhai, GS, Hu, B, Yasuda, K, Michel, A: Qualitative analysis of discrete-time switched systems. In: Proc of the American Control Conference. 1880-1885 (2002)

14. Zhang, WA, Li, Yu: Stability analysis for discrete-time switched time-delay systems. Automatica. 45, $2265-2271$ (2009). doi:10.1016/j.automatica.2009.05.027

15. Uhlig, F: A recurring theorem about pairs of quadratic forms and extensions. Linear Algebra Appl. 25, $219-237$ (1979)

16. Agarwal, RP: Difference Equations and Inequalities, Oth edn.Marcel Dekker, New York (2000)

17. Khusainov, DYa, Diblik, J, Svoboda, Z, Smarda, Z: Instable Trivial Solution of Autonomous Differential Systems with Quadratic Right-Hand Sides in a Cone. Abstract and Applied Analysis (2011). Article ID 154916, 23 pages

18. Diblik, J, Khusainov, DYa, Grytsay, IV, Smarda, Z: Stability of Nonlinear Autonomous Quadratic Discrete Systems in the Critical Case. Discrete Dynamics in Nature and Societe (2010). Article ID 539087, 23 pages

19. Diblik, J, Khusainov, DYa, Ruzickova, : Solutions of Discrete equations with prescrid asymptotic behavior. Dynamic Systems and Applications. 4, 395-402 (2004)

20. Diblik, J, Khusainov, DYa, Grytsay, Irina V: Stability Investigation of Nonlinear Quadratic Discrete Dynamics Systems in the Critical Case. International Symposium on Nonlinear Dynamics, Journal of Physics: Conference Series 96. IOP Publishing (2008)

21. Bastinec, J, Diblik, J, Khusainov, DYa, Ryvolova, A: Exponential Stability and Estimation of Solutions of Linear Differential Systems of Neutral Type with Constant Coefficients. Boundary Value Problems (2010). Article ID 956121, 20 pages

22. Diblik, J, Hlavickova, I: Combination of Liapunov and Retract Methods in the Investigation of the Asymptotic Behavior of Solutions of Systems of Discrete Equations. Dynamic Systems and Applications. 18, 507-538 (2009)

23. Bastinec, J, Diblik, J, Smarda, Z: Existence of positive solutions of discrete linear equations with a single delay. Journal of Diference Equations and Applications. 9, 1047-1056 (2010)

24. Diblik, J, Ruzickova, M, Smarda, Z: Wazewskis method for systems of dynamic equations on time scales. Nonlinear Analysis, Theory, Methods and Applications. 71, 1124-1131 (2009). doi:10.1016/j.na.2008.11.027

doi:10.1186/1687-1847-2012-28

Cite this article as: Sangapate: New sufficient conditions for the asymptotic stability of discrete time-delay systems. Advances in Difference Equations 2012 2012:28. 